\title{
Influência da temperatura na síntese hidrotérmica da zeólita Y obtida por microondas
}

\section{(Influence of temperature on the hydrothermal synthesis of zeolite Yobtained by microwave)}

\author{
A. N. Simões ${ }^{1}$, L. S. Neiva ${ }^{1}$, V. N. Simões ${ }^{1}$ M. G. Rodrigues ${ }^{2}$ L. Gama ${ }^{1}$ \\ ${ }^{1}$ Unidade Acadêmica de Engenharia de Materiais, ${ }^{2}$ Departamento de Engenharia Química \\ Universidade Federal de Campina Grande, Av. Aprígio Veloso, 882, Bodocongó, 58109-970, \\ Campina Grande, PB, Brasil \\ alluskynha@hotmail.com
}

\begin{abstract}
Resumo
Nos últimos anos o aquecimento e a condução de reações químicas por energia de microondas tem sido um tema cada vez mais popular na comunidade científica, e assim nos campos da zeólita. Desta forma, o presente trabalho visa estudar o efeito da temperatura na obtenção da zeólita Y sintetizada por microondas. A temperatura de síntese foi variada de $100{ }^{\circ} \mathrm{C}$ a $90{ }^{\circ} \mathrm{C}$. As amostras foram caracterizadas por difração de raios X, microscopia eletrônica de varredura e adsorção de nitrogênio. Os resultados comprovaram a forte influência da temperatura em todas as características finais das amostras sintetizadas, sendo a zeólita Y obtida a $90{ }^{\circ} \mathrm{C}$ por microondas. A temperatura de $100{ }^{\circ} \mathrm{C}$ foi a ideal para a obtenção da zeólita com boa cristalinidade.

Palavras-chave: zeólita $\mathrm{Y}$, microondas.
\end{abstract}

\begin{abstract}
In recent years, heating and driving chemical reactions by microwave energy has been an increasingly popular topic in the scientific community, and so in the field of zeolite. Thus, this paper aims to study the effect of temperature on the achievement of zeolite $Y$ synthesized by microwave. The synthesis temperature was varied from $100{ }^{\circ} \mathrm{C}$ to $90^{\circ} \mathrm{C}$. The samples were characterized by X-ray diffraction, scanning electron microscopy and nitrogen adsorption. The results show the strong influence of temperature on all the final characteristics of the samples. As the Y zeolite obtained at $90{ }^{\circ} \mathrm{C}$ by microwave, but $100{ }^{\circ} \mathrm{C}$ was optimal temperature for obtaining the zeolite with good crystallinity.
\end{abstract}

Keywords: zeolite Y, microwave.

\section{INTRODUÇÃO}

As microondas são ondas eletromagnéticas nãoionizantes, com freqüências entre $300 \mathrm{MHz}$ e $30 \mathrm{GHz}$, as quais correspondem a comprimentos de onda de $1 \mathrm{~mm}$ a $1 \mathrm{~m}$ [1]. O aquecimento por microondas é também chamado de aquecimento dielétrico, e existem dois mecanismos principais para a transformação de energia eletromagnética em calor [2]. O primeiro deles é chamado rotação de dipolo, e relaciona-se com o alinhamento das moléculas (que tem dipolos permanentes ou induzidos) com o campo elétrico aplicado. Estes movimentos das moléculas excitadas perturbam as outras moléculas, não excitadas, de modo que o conjunto é aquecido através das colisões geradas. $\mathrm{O}$ segundo mecanismo é chamado de condução iônica, e o calor é gerado através de perdas por friç̧ão, que acontecem pela migração de íons dissolvidos quando sob a ação de um campo eletromagnético. Durante o aquecimento dielétrico, as radiações penetram no material de forma que a transferência de calor acontece desde o interior do material até a superfície. Este tipo de transferência causa o aquecimento em massa do material e um rápido aumento da sua temperatura. Desta forma, este tipo de aquecimento é bem diferente do convencional, no qual as taxas de aquecimento são mais lentas e dependem da condutividade térmica do material, das diferenças de temperatura criadas ao longo do material e das correntes convectivas [3]. Recentemente, foi relatado que o método hidrotérmico assistido por microondas poderia fornecer uma eficiente maneira de sintetizar rapidamente vários cristais de zeólita e racionalmente controlar sua distribuição de tamanho de partículas [4-6], a pureza da fase [7] e morfologia [8]. Como uma espécie de fonte de energia nova, microondas foi sugerida para alterar a cinética e seletividade das reações de forma otimizada [9], o que reduziu significativamente o tempo de síntese de vários dias a alguns minutos [10].

A síntese de zeólitas é conhecida por ser um processo complexo em que todos os parâmetros de síntese, incluindo composição do gel, temperatura, tempo e natureza dos materiais de partida contribuem de uma forma específica para o processo de cristalização. Dessa forma, o objetivo deste trabalho é sintetizar a zeólita Y pelo método hidrotérmico 
assistido por microondas e estudar o efeito da temperatura nas características finais da zeólita.

\section{MATERIAIS E MÉTODOS}

Para a preparação da zeólita $\mathrm{Y}$ foram utilizados os seguintes reagentes: sílica $\left(\mathrm{SiO}_{2}\right)$ amorfa, hidróxido de sódio $(\mathrm{NaOH})$, aluminato de sódio $\left(\mathrm{Na}_{2} \mathrm{Al}_{2} \mathrm{O}_{2}\right)$ e água destilada. A síntese foi realizada da seguinte forma: preparou-se uma primeira solução, denominada solução $A$, com $\mathrm{NaOH}, \mathrm{SiO}_{2}$ e água destilada e então deixou a solução envelhecendo sob agitação constante por $4 \mathrm{~h}$. Em seguida, preparouse uma segunda solução, denominada solução $B$, com $\mathrm{NaOH}, \mathrm{Na}_{2} \mathrm{Al}_{2} \mathrm{O}_{2}$ e água destilada. Adicionou-se a solução $B$ lentamente na solução $A$, homogeneizando por meio de agitação mecânica à temperatura ambiente, durante mais $4 \mathrm{~h}$. Dessa forma, foi obtido um gel, que permaneceu em repouso durante $24 \mathrm{~h}$, quando ocorreu o envelhecimento. Depois deste período, o gel de síntese foi colocado no equipamento de microondas (Shimadzu) variando a temperatura, sendo estas de $100{ }^{\circ} \mathrm{C}$ a $90{ }^{\circ} \mathrm{C}$, durante $60 \mathrm{~min}$. Concluído o tratamento hidrotérmico, a amostra foi centrifugada até o $\mathrm{pH}$ tornar-se neutro. Em seguida, a amostra foi seca na estufa a $60{ }^{\circ} \mathrm{C} / 24 \mathrm{~h}$. Após esse processo, as amostras foram trituradas em um almofariz e peneiradas para posterior caracterização. Os pós resultantes foram caracterizados quanto à determinação das fases formadas, o tamanho de cristalito e a cristalinidade a partir dos dados de difração de raios X em difratômetro Shimadzu 6000, radiação Cuk, 40 kV e 30 mA. Para identificação das fases utilizou-se o programa (Pmgr) Shimadzu e o banco de dados JCPDF. A cristalinidade foi obtida no programa (Pmgr) Shimadzu Cristalinity com coeficiente de correção de Lorentz, no qual foi possível calcular o percentual de fase cristalina. E o tamanho médio de cristalito foi calculado a partir do alargamento dos picos de reflexão basal usando a equação de Scherrer [11]. A área superficial específica foi determinada por adsorção de nitrogênio, com a técnica desenvolvida por Brunauer, Emmett e Teller (BET) (Micromeritics Gemini-2370) e os aspectos morfológicos dos pós foram analisados por meio de microscopia eletrônica de varredura em um microscópio Philips XL30 FEG.

\section{RESULTADOS E DISCUSSÃO}

$\mathrm{O}$ efeito da temperatura na produção da zéolita $\mathrm{Y}$ é apresentado a seguir. As Figs. 1 e 2 exibem os difratogramas de raios $\mathrm{X}$ das amostras sintetizadas em $60 \mathrm{~min}$ a $100^{\circ} \mathrm{C}$ e $90{ }^{\circ} \mathrm{C}$, respectivamente. Estas amostras foram denominadas ZYM1 e ZYT1.

As reflexões são características da estrutura da zeólita $\mathrm{Y}$ (JCPDS 43-0168), de $2 \theta=5$ a $35^{\circ}$, confirmando a formação da zeólita Y. Vale ressaltar que o tempo de síntese necessário para obtenção da zeólita pelo método assistido por microondas $(60 \mathrm{~min})$ foi bem inferior ao tempo de síntese para a obtenção da mesma zeólita, usando o método hidrotérmico convencional [12], sendo esta diminuição de

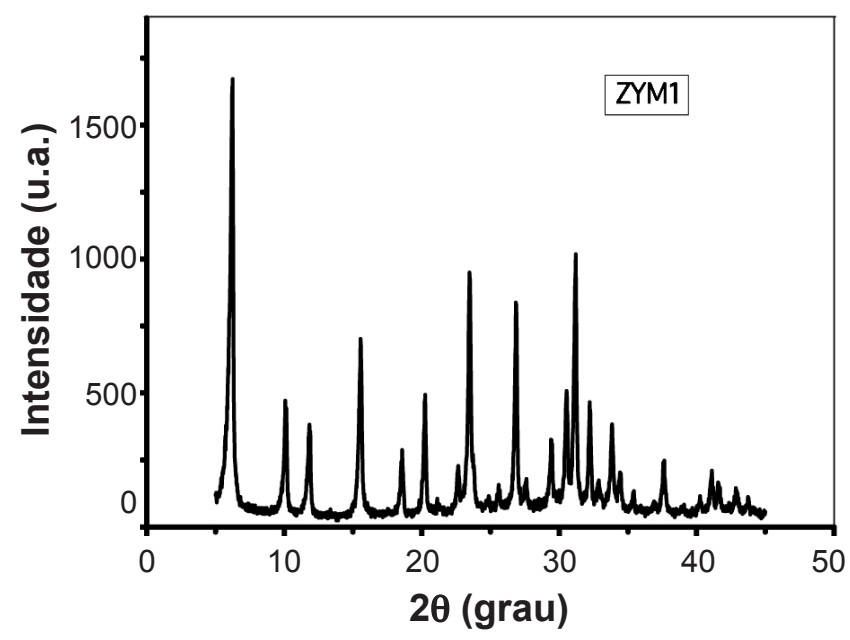

Figura 1: Difratograma de raios $\mathrm{X}$ da zeólita $\mathrm{Y}$ obtida por síntese hidrotérmica assistida por microondas a $100^{\circ} \mathrm{C}$.

[Figure 1: XRD pattern of zeolite $Y$ obtained by hydrothermal synthesis assisted by microwave at $100^{\circ} \mathrm{C}$.]

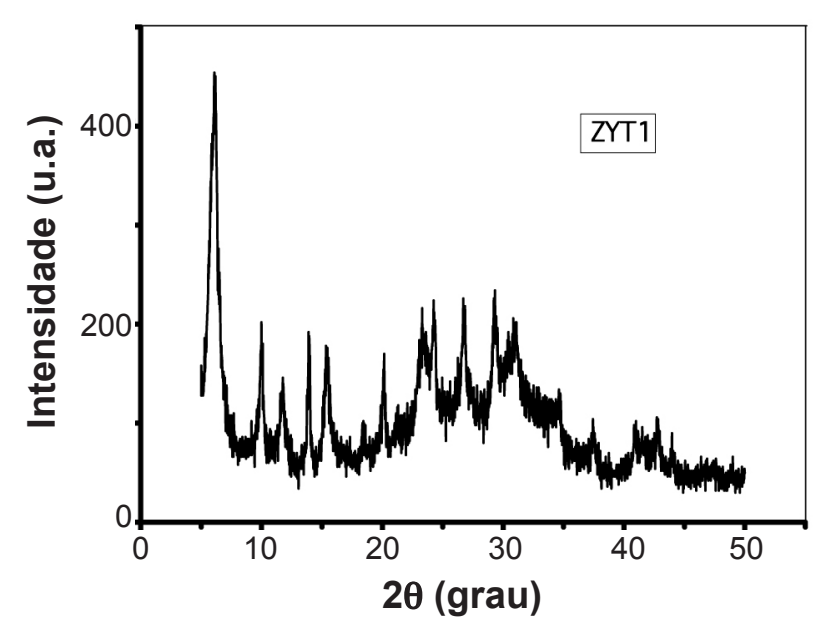

Figura 2: Difratograma de raios $\mathrm{X}$ da zeólita $\mathrm{Y}$ obtida por síntese hidrotérmica assistida por microondas a $90^{\circ} \mathrm{C}$.

[Figure 2: XRD pattern of zeolite $Y$ obtained by hydrothermal synthesis assisted by microwave at $90^{\circ} \mathrm{C}$.]

$72 \mathrm{~h}$ para $1 \mathrm{~h}$. E esta redução é uma das maiores vantagens da tecnologia de microondas. Isto porque as microondas interagem diretamente com o gel, em contraste com as rotas convencionais onde o aquecimento é feito através da condução e convecção [13]. A Fig. 2 mostra a formação da zeólita Y com a diminuição da temperatura de $100{ }^{\circ} \mathrm{C}$ para $90^{\circ} \mathrm{C}$. Porém, foi observada também a existência de material amorfo com a diminuição de temperatura, com um largo pico amorfo em $2 \theta=20$ a $35^{\circ}$ em conjunto com alguns picos característicos da zeólita Y. A diminuição da cristalinidade com a diminuição da temperatura se deve ao fato de que em temperaturas mais baixas o processo de cristalização ocorre em uma menor taxa, sendo necessário então um maior tempo de reação para a formação da zeólita Y com boa cristalinidade, em temperaturas inferiores a $100{ }^{\circ} \mathrm{C}$, comprovando assim, que neste estudo, na obtenção da zeólita $\mathrm{Y}$, tempo e temperatura estão 
diretamente relacionados. A influência da temperatura e do tempo na obtenção da fase ZSM-5 sintetizada por aquecimento convencional foi estudada [14]. Primeiro, o tempo de reação foi fixado em $24 \mathrm{~h}$, enquanto a zeólita foi exposta a $130,150,170$ e $200{ }^{\circ} \mathrm{C}$. Foi verificado que em $130{ }^{\circ} \mathrm{C}$ apenas material amorfo foi formado, sendo a temperatura de $150{ }^{\circ} \mathrm{C}$ a que obteve melhor cristalinidade da zeólita. Em seguida a temperatura de $150{ }^{\circ} \mathrm{C}$ foi fixada e o tempo variado de $12,14,16,18,20,22$ e $24 \mathrm{~h}$ e foi visto que é necessário um tempo mínimo de $18 \mathrm{~h}$ para a formação da zeólita, sendo o tempo mais longo, de 24 h, o que levou a uma maior cristalinidade. Foi mostrado que a ZSM-5 sintetizada por meio de microondas pode ser conseguida com menor tempo de aquecimento em maiores temperaturas [15]. Para estudar o efeito da temperatura, a mesma foi variada de $120{ }^{\circ} \mathrm{C}$ a $180{ }^{\circ} \mathrm{C}$. A $120{ }^{\circ} \mathrm{C} / 10 \mathrm{~h}$, apenas material amorfo foi obtido, porém ao se prolongar esse tempo para $20 \mathrm{~h}$, a quantidade de material amorfo diminuiu e cristais de ZSM-5 foram observados. E quando a temperatura foi elevada para $180^{\circ} \mathrm{C}$, os cristais de ZSM5 foram observados em um tempo mais curto, $10 \mathrm{~h}$.

A Tabela I exibe os dados de tamanho de cristalito das amostras ZYM1 e ZYT1.

Tabela I - Tamanho de cristalito e cristalinidade das amostras ZYM1 e ZYT1.

[Table I - Crystallite size and crystallinity of the samples and ZYM1 ZYT1.]

\begin{tabular}{ccc}
\hline Amostras & $\begin{array}{c}\text { Tamanho de } \\
\text { cristalito }(\mathrm{nm})\end{array}$ & $\begin{array}{c}\text { Cristalinidade } \\
(\%)\end{array}$ \\
\hline ZYM1 & 29,32 & 82,6 \\
ZYT1 & 10,94 & 50,0 \\
\hline
\end{tabular}

Analisando as amostras ZYM1 e ZYT1 sintetizadas por microondas, verifica-se que quando a temperatura é diminuída de $100{ }^{\circ} \mathrm{C}$ para $90{ }^{\circ} \mathrm{C}$, o tamanho do cristalito diminui de $29,3 \mathrm{~nm}$ para $10,9 \mathrm{~nm}$, ou seja, os cristais de maior tamanho foram obtidos em maior temperatura $\left(100{ }^{\circ} \mathrm{C}\right)$, mostrando que o tamanho do cristal depende fortemente da temperatura de síntese. $O$ fato da cinética de crescimento dos cristais ser dependente da temperatura é explicado pelo mecanismo de nucleação autocatalítica, onde temperaturas mais altas vão acelerar a velocidade de dissolução do gel e causar o crescimento de numerosos núcleos mais rápido [16]. Além disso, acredita-se que a temperatura mais baixa favorece a nucleação, enquanto o crescimento do cristal irá superar o processo de nucleação em temperaturas mais elevadas. Foi também mostrada essa relação do tamanho do cristal com a temperatura [17]. Foi sintetizada a zeólita beta, variando a temperatura de síntese de 80 a $160{ }^{\circ} \mathrm{C}$. Nesse estudo, foi observado, como conseqüência do aumento da temperatura, um aumento no tamanho dos cristalitos da zeólita, que variou de 43,7 a $88,4 \mathrm{~nm}$. Ao ser sintetizada a zeólita $\mathrm{L}$ via microondas variando a temperatura de $170{ }^{\circ} \mathrm{C}$ a $190{ }^{\circ} \mathrm{C}$, também
Tabela II - Área superficial das amostras ZYM1 e ZYT1. [Table II - Surface area of samples ZYM1 and ZYT1.]

\begin{tabular}{cc}
\hline Amostras & $\begin{array}{c}\text { Área superficial } \\
\left(\mathrm{m}^{2} / \mathrm{g}\right)\end{array}$ \\
\hline ZYM1 & 476,2 \\
ZYT1 & 344,9 \\
\hline
\end{tabular}

foi relatado o aumento do tamanho dos cristalitos com a elevação da temperatura de síntese [18]. A cristalinidade da zeólita $\mathrm{Y}$ diminuiu de $82,6 \%$ para $50 \%$ com a redução da temperatura de $100{ }^{\circ} \mathrm{C}$ para $90{ }^{\circ} \mathrm{C}$. Assim, a melhor condição apontada por este trabalho para a síntese da zeólita Y via microondas é usando $100{ }^{\circ} \mathrm{C} / 60 \mathrm{~min}$.

A Tabela II mostra valores de área superficial das amostras ZYM1 e ZYT1.

Com a diminuição da temperatura de síntese de $100{ }^{\circ} \mathrm{C}$ para $90{ }^{\circ} \mathrm{C}$ os valores de área superficial também se alteram de $476 \mathrm{~m}^{2} / \mathrm{g}$ para $345 \mathrm{~m}^{2} / \mathrm{g}$. Isto ocorre porque a amostra sintetizada na menor temperatura (ZYT1) apresentou uma maior quantidade de material amorfo, de acordo com os resultados de DRX. Ao se estudar a síntese da zeólita Y usando caulim como fonte de silício, também foi observada a diminuição da área superficial da zeólita Y, quando a temperatura de cristalização foi diminuida [19].

As Figs. 3 e 4 exibem a morfologia obtida por microscopia eletrônica de varredura das amostras sintetizadas por microondas a $100{ }^{\circ} \mathrm{C}(Z Y M 1)$ e $90{ }^{\circ} \mathrm{C}$ (ZYT1), respectivamente.

As partículas apresentam uma morfologia octaédrica bem definida, típica da zeólita Y [20]. As partículas também

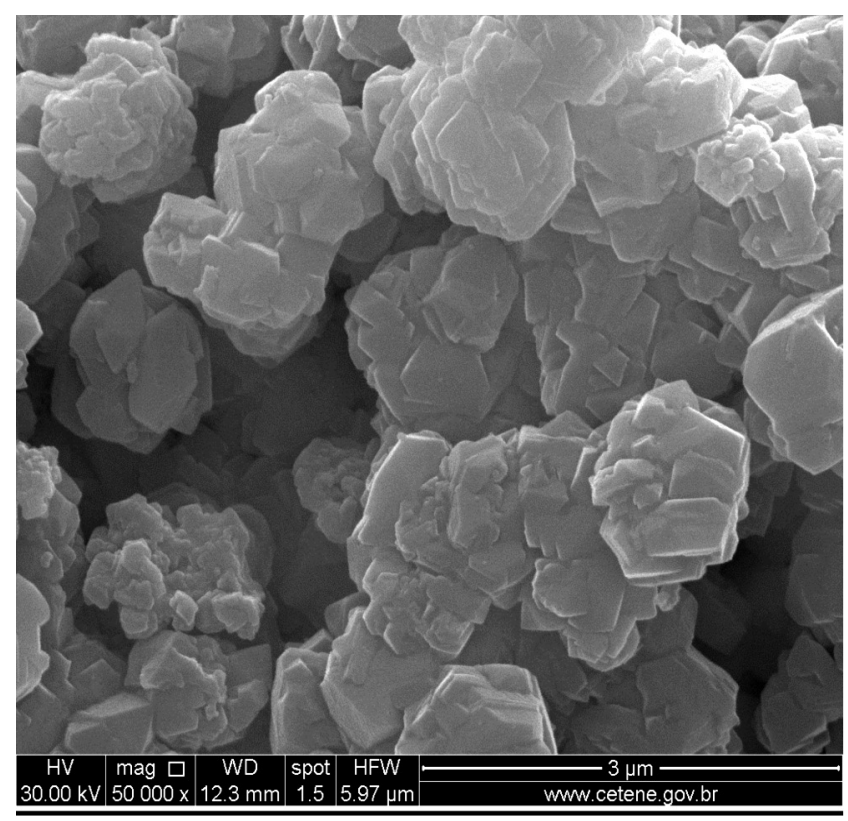

Figura 3: Micrografia obtida por microscopia eletrônica de varredura da amostra ZYM1.

[Figure 3: Scanning electron microscopy micrograph of the sample ZYM1.] 


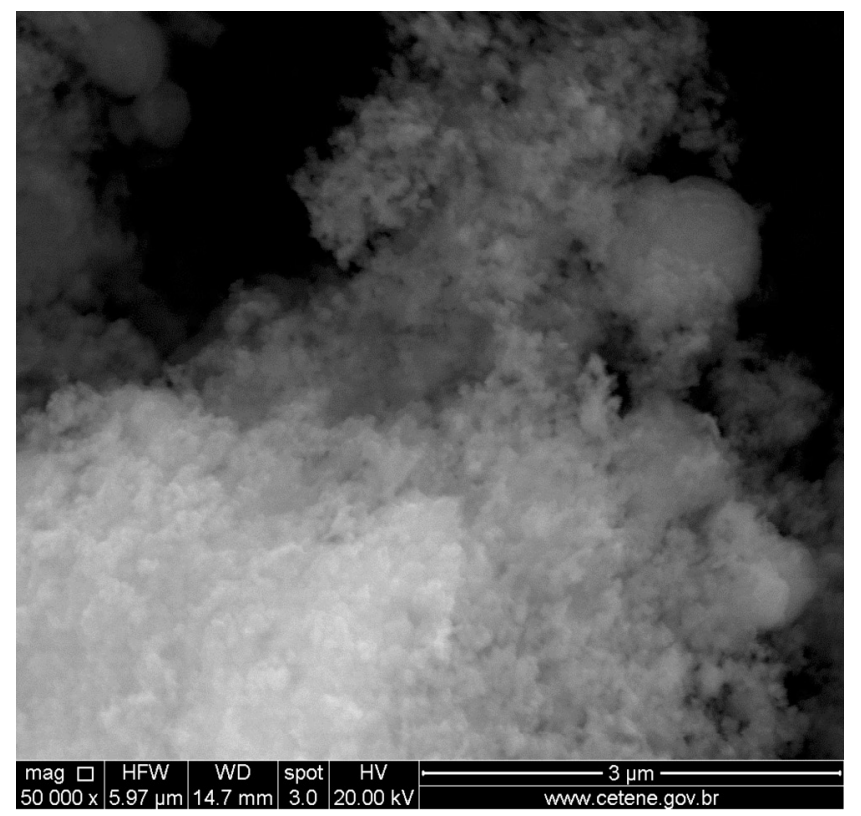

Figura 4: Micrografia obtida por microscopia eletrônica de varredura da amostra ZYT1.

[Figure 4: Scanning electron microscopy micrograph of the sample ZYT1.]

estão aglomeradas, porém com uma distribuição estreita de partículas, devido ao crescimento simultâneo dos núcleos, que claramente prevaleceu sobre a nucleação. Logo, as amostras de microondas são bem definidas e uniformes. De acordo com a micrografia apresentada na Fig. 4, não foi possível observar a formação de nenhuma partícula referente a zeólita $\mathrm{Y}$, mas apenas quantidades significativas de material amorfo, o que está de acordo com os resultados da difração de raios X, nos quais a amostra ZYT1 apresenta bandas características de material amorfo.

\section{CONCLUSÕES}

O método de síntese hidrotérmica assistida por microondas pode ser recomendado como um método promissor, visto que a zeólita Y foi obtida com sucesso. A zeólita $\mathrm{Y}$ foi obtida na temperatura mínima de $90{ }^{\circ} \mathrm{C}$ por microondas, porém $100{ }^{\circ} \mathrm{C}$ é a temperatura ideal para a obtenção da zeólita com boa cristalinidade. Com a diminuição da temperatura de síntese, o tamanho dos cristais e a área superficial diminuíram. Através da microscopia eletrônica de varredura foi possível verificar que as partículas são bem definidas e uniformes, quando sintetizadas a $100{ }^{\circ} \mathrm{C}$, porém a $90{ }^{\circ} \mathrm{C}$ apenas quantidades significativas de material amorfo foram observadas, o que mostra que a temperatura altera todas as características finais da zeólita Y sintetizada por microondas.

\section{AGRADECIMENTOS}

Os autores agradecem a CAPES pelo financiamento e suporte destinados a esta pesquisa.

\section{REFERÊNCIAS}

[1] A. M. Sanseverino, Química Nova 25 (2002) 660.

[2] H. M. Kingston, L. B. Jassie, Introduction to Microwave Sample Preparation, ACS Prof. Ref. Book, Washington D.C., EUA (1988) 93.

[3] M. Fortuny, A. L. D. Ramos, C. Dariva, S. M. S. Egues, A. F. Santos, Química Nova 31 (2008) 1553.

[4] S. Komarneni, R. Roy, Q. H. Li, Mater. Res. Bull. 27 (1992) 1393.

[5] C. S. Cundy, Collect. Czech. Chem. Comm. 63 (1998) 1699.

[6] C. S. Cundy, J. O. Forrest, Micropor. Mesopor. Mater. 72 (2004) 67.

[7] S. H. Jhung, J. S. Chang, J. S. Hwang, S. E. Park, Micropor. Mesopor. Mater. 64 (2003) 33.

[8] J. C. Lin, J. T. Dipre, M. Z. Yates, Langmuir 20 (2004) 1039.

[9] G. A. Tompsett, W. C. Conner, K. S. Yngvesson, Chem. Phys. Chem. 7 (2006) 296.

[10] A. N. Simões, Síntese de Zeólita Y via tratamento hidrotérmico convencional e assistido por microondas, Diss. Mestrado em Ciência e Engenharia de Materiais, UFCG, PB (2011).

[11] H. Klug, L. Alexander, X- ray diffraction procedures, Wiley, New York (1962) 491.

[12] A. P. Araújo, Síntese de membrana zeolítica (zeólita Y/ membrana cerâmica) visando sua aplicação na catálise, Diss. Mestrado em Engenharia Química, UFCG, PB (2010).

[13]A. E. Taylor, Microwave synthesis and occlusion reactions of zeolites, Tese, Univ. Birmingham, Inglaterra (2007).

[14] R. Anuwattana, K. J. Balkus Jr., S. Asavapisit, P. Khummongkol, Micropor. Mesopor. Mater. 111 (2008) 260.

[15] P. Phiriyawirut, R. Magaraphan, A. M. Jamieson, S. Wongkasemjit, Mater. Sci. Eng. A 361 (2003) 147.

[16] Y. Hu, C. Liu, Y. Zhang, N. Ren, Y. Tang, Micropor. Mesopor. Mater. 119 (2009).

[17] B. Modhera, M. Chakraborty, P. A. Parikh, R. V. Jasra, Cryst. Res. Technol. 44 (2009) 379.

[18] M. Holzl, S. Mintova, T. Bein, Stud. Surf. Sci. Catal. 158 (2005) 11.

[19] X. Liu, Z. Yan, H. Wang, Y. Luo, J. Natural Gas Chem. 12 (2003) 63.

[20] V. Valtchev, S. Rigolet, K. N. Bozhilov, Micropor. Mesopor. Mater. 101 (2007) 73.

(Rec. 13/12/2011, Ac. 30/03/2012) 